\title{
CORRELATION LENGTH IN PBnA LIQUID CRYSTAL FAMILY
}

\author{
K. El Guermai ${ }^{a, b *}$, M. Ayadi ${ }^{a}$ AND K. EL Boussiri ${ }^{b}$ \\ ${ }^{a}$ Département de Physique, Faculté des Sciences \\ route d'El Jadida, Casablanca, Maroc \\ ${ }^{b}$ Faculté de Médecine Dentaire \\ rue Abou Al Aala Zahar, B.P. 9157, Casablanca, Maroc \\ (Received July 6, 1998; in final form November 4, 1998)
}

\begin{abstract}
We present a study of the range molecular interactions inside layers in smectic phases A and I of PBnA (phenyl-4 benzylidene 4'-alkylaniline) liquid crystals family. The purpose of this work is to determine the correlation length of the molecules' gravity centers in existing domain of smectic phases and at the transition between them. We used X-rays in-plane peaks of the pattern diffraction of aligned sample by a magnetic field.

PACS numbers: 61.30.-v, 61.10.-i, 64.70.Md, 61.30.Gd
\end{abstract}

\section{General}

The notion of correlation length [1-4] is easy to understand if we take into consideration the fluidity of liquid crystals. It is clear that this property is incompatible with infinite range of translation order. If we are placed on a specific point of smectic plane, the position of neighboring molecules will be entirely determined by the basic transition of smectic order. However this translation order is lost if we consider further molecules. The length at which this disorder becomes predominant is called correlation length. This correlation length can be taken in a simple way as the particle's ray having two-dimensional crystalline order that constitutes the smectic plan. This order was previously understood in the framework of the Nelson-Halperin theory [5] based itself upon the Kosterlitz-Thouless theory [6]. For these authors the melting of two-dimensional solid is assisted by continued dissociation of dislocation pairs. But recent results of high-resolution heat-capacity study and electron diffraction measurements performed on a number of liquid crystalline series in the vicinity of the smectic A-smectic I (SA-SI) phase transition have shown [7-10] an important disagreement with the predictions of Nelson-Halperin theory.

To participate to this debate, we havestudied and determined, by X-rays, the correlation length of the position order of molecules inside layers in smectic phases $\mathrm{A}$ and I of family PBnA of liquid crystals (phenyl-4 benzylidene 4 '-alkylaniline).

\footnotetext{
*Corresponding author.
} 


\section{Experimental setup}

For X-ray measurements, the samples of $\mathrm{PBnA}(n=8,10,12$, and 14$)$ liquid crystals were put in a magnetic field and heated to the nematic (or isotropic) phase. The temperature was then lowered to the smectic phase and the field turned off.

We have used the same spectrometer as described in detail in [11-13]. The incoming X-ray beam ( $\left.\mathrm{Cu} K_{\alpha}, 40 \mathrm{kV}, 20 \mathrm{~mA}, \lambda_{K_{\alpha}}=1.54059 \AA\right)$ is monochromatized by the (1011) Bragg reflection of a curved quartz monochromator; its divergence is reduced by a set of vertical and horizontal slits which are also able to strip the $K_{\alpha_{2}}$ line. The liquid crystal sample was introduced into a Lindemann glass capillary of diameter $0.7 \mathrm{~mm}$ and 5 to $8 \mathrm{~mm}$ long placed vertically in a goniometer head. The capillary can be continuously rotated around the vertical axis of the goniometer in order to average over all orientations perpendicular to the rotation axis. The scattered beam was detected by a curved linear position sensitive multidetector (INEL CPS 120) of the gas flow type, which covers $120^{\circ}$ symmetrically relative to the main beam.

The sample was pliced in its center. The X-ray photons scattered by the sample in the equatorial plane were measured with an electronic resolution of about $0.03^{\circ}$, the window height being $8 \mathrm{~mm}$. A beam stop allowed stripping of the incident beam. The gas flux and pressure, and the high voltage, discrimination of the incoming pulses were controlled by electronic devices, allowing through delay lines, the determination of the position and the intensity of the detected photons. The obtained data was stored in a $4 \mathrm{~K}$ multichannel analyzer with visualization facilities and the possibilities for treatment. The instrumental width was about 5 channels $\left(0.15^{\circ}\right)$ FWHM with nearly Gaussian incoming beam shape. The diffraction patterns were then stored in a personal computer which was also used to pilot data collections.

The sample was introduced into a gas flow cryostat [14] and could be rotated from the top of the cryostat. Mylar windows made it possible minimization of the attenuation of the incoming and scattered beams and their aperture allowed us to use the curved detector over all its angular range. The temperature was controlled to about $0.1 \mathrm{~K}$. A magnetic field up to $0.8 \mathrm{~T}$ was used to align samples.

\section{Results and discussion}

It is well known that influence of disorder over the diffraction pattern results essentially in an enlargement of Bragg's peaks. The procedure to obtain correlation length from this enlargement was already done by many authors [15-17]. To interpret the shape of this type of peaks, we followed theoretical considerations that already regrouped (see Ref. [18]). It is shown that to study the short-range position order, the experimental profile is to be adjusted by the Lorentzian form. By choosing this form, we obtain good correspondence between experimental profile and the calculated one.

Figures 1 represent experimental and calculated profiles of diffraction rays with large angle in the following phases: SI (a) and SA (b) in the PB10A selected as a typical example in the $\mathrm{PBnA}$ family. The deconvolution of experimental peaks and their adjustment is fitted by the "Curve-Fit" program. The enlargement of 

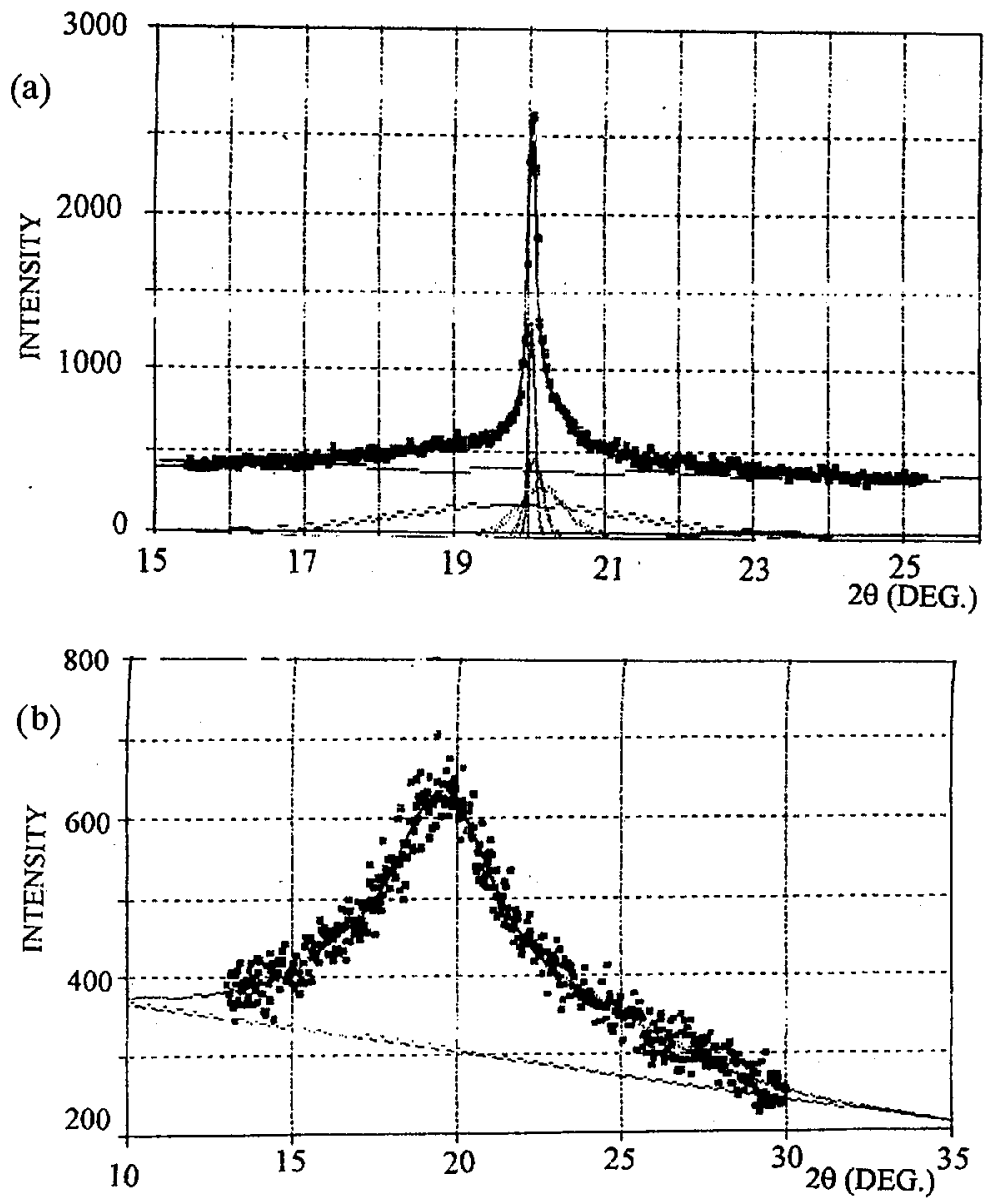

Fig. 1. Typical X-ray diffraction patterns for PBnA family. PB10A taken as an example: (a) smectic I, (b) smectic A.

peaks in the smectic phase A corresponds to the diffusion of the molecules gravity centers, consequently, to the loss of the correlation of position order inside smectic layers.

Before examining the results, a reminder of the polymorphism, the transition temperatures and the enthalpy variations for different homologues of $\mathrm{PBnA}$ series is in the following table (Table I [10]).

Figures 2-5 represent variation of correlation length of position order inside layers, calculated from the patterns profiles from diffraction $\mathrm{X}$ with respect to temperature for homologous PBnA ( $n=8,10,12$, and 14).

The short-range position order in the smectic phase $A(\xi \approx 22 \AA)$ changes abruptly in the transition to tilted hexatic smectic phase $I(\xi \approx 162 \AA)$. Within this last phase, $\xi$ increases in continuous fashion up to $\approx 190 \AA$ for PB8A and PB10A and $\approx 240 \AA$ for other homologues. However, the correlation of the position order inside the smectic layer $I$ is not saturated. On the contrary, it is increasing when 
TABLE

Temperature $T\left({ }^{\circ} \mathrm{C}\right)$ and enthalpy $\Delta H$ (kcal/mol) of different phase transitions (Cr-SI, SI-SA, SA-N or SA-Is and N-Is) for $\mathrm{PBnA}(n=8,10,12$, and 14) liquid crystal family. $\mathrm{N}$ - nematic, Is - isotropic, $\mathrm{Cr}$ crystal.

\begin{tabular}{|c|c|c|c|c|}
\hline$n$ & $\mathrm{Cr}-\mathrm{SI}$ & SI-SA & $\mathrm{SA}-\mathrm{N}$ & $\mathrm{N}-\mathrm{Is}$ \\
\hline$T$ & 89.55 & 125.81 & 127.92 & 130.73 \\
\hline \multicolumn{5}{|l|}{8} \\
\hline$\Delta H$ & 10.63 & 2.61 & 0.52 & 0.88 \\
\hline$T$ & 96.25 & 122.81 & 128.54 & \\
\hline 10 & & & & - \\
\hline$\Delta H$ & 9.86 & 2.28 & 0.88 & \\
\hline$T$ & 100.6 & 116.08 & 122.32 & \\
\hline 12 & & & & - \\
\hline$\Delta H$ & 10.66 & 2.25 & 0.96 & \\
\hline$T$ & 104.56 & 113.42 & 121.45 & \\
\hline 14 & & & & - \\
\hline$\Delta H$ & 11.44 & 2.24 & 1.041 & \\
\hline
\end{tabular}

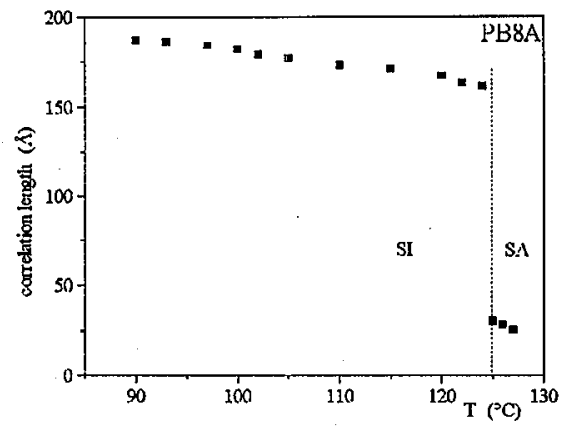

Fig. 2. Temperature dependence of the correlation length of the order calculated from $\mathrm{X}$-ray diffraction for PB8A compound.

temperature is decreasing. The same variation sign is observed in the variations of the tilt angle for the same compounds. These facts show uniquely the coupling and the intense competition between these two orders from SA to SI transition and continue up to crystallization.

It is also noted that in the smectic I phase, the absolute value of the slopes $(\Delta \xi / \Delta T)$ are very different. These values are approximately equal to $1 \AA /{ }^{\circ} \mathrm{C}$ for $\mathrm{PB} 8 \mathrm{~A}$ and $\mathrm{PB} 10 \mathrm{~A}$, to $6 \AA /{ }^{\circ} \mathrm{C}$ for $\mathrm{PB} 12 \mathrm{~A}$ and to $10 \AA /{ }^{\circ} \mathrm{C}$ for PB14A. 


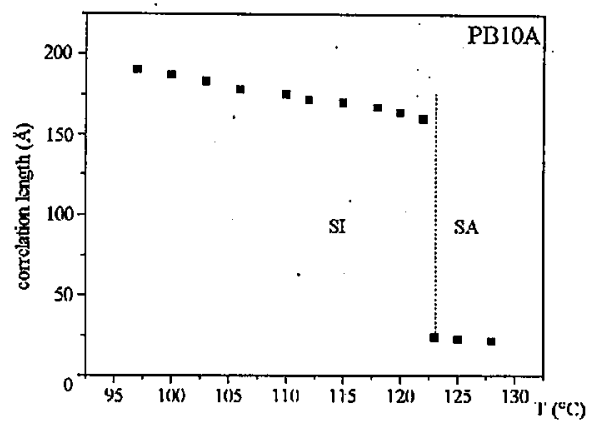

Fig. 3. Temperature dependence of the correlation length of the order calculated from $\mathrm{X}$-ray diffraction for $\mathrm{PB} 10 \mathrm{~A}$ compound.

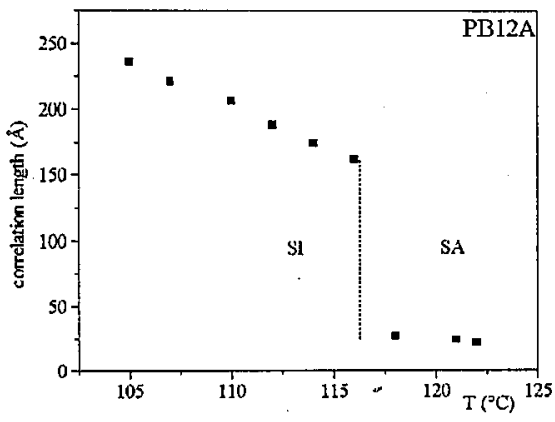

Fig. 4. Temperature dependence of the correlation length of the order calculated from $\mathrm{X}$-ray diffraction for PB12A compound.

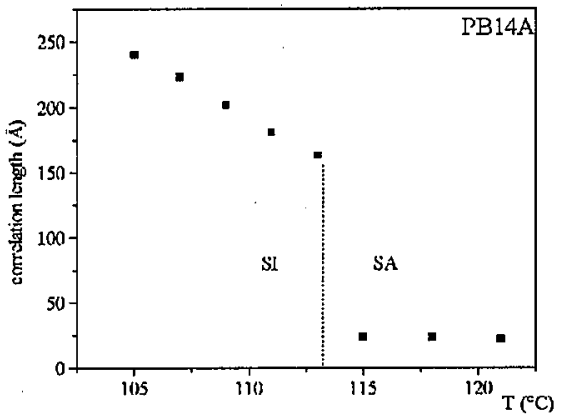

Fig. 5. Temperature dependence of the correlation length of the order calculated from $\mathrm{X}$-ray diffraction for PB14A compound.

\section{Conclusion}

In this work, we have answered the question previously put forward in Ref. [10]; we have evaluated the range of molecular interactions, inside smectic layers. 
Next important step, is to collect together the set of all data concerning PBnA: interlayer distance, the molecular tilt angle, and the in-layer correlation length in order to extract a phenomenological model to put into perspective the coupling between different order parameters.

\section{Acknowledgments}

We are grateful to Centre International des Etudiants et Stagiaires for financial support (contract $201274 \mathrm{~K}$ ). We thank also Drs. Carole Gors and Vladimir Lorman for the technical assistance and helpful discussion.

\section{References}

[1] J.J. Benattar, A.M. Levelut, M. Lambert, J. Phys. (France) 41, 1371 (1980).

[2] P.A.C. Gane, A.J. Leadbetter, J.J. Benattar, F. Moussa, M. Lambert, Phys. Rev. A 24, 2694 (1981).

[3] A.M. Levelut, J. Doucet, J.J. Benattar, M. Lambert, B. Dorner, J. Phys. (France) 42, 1651 (1981).

[4] J.J. Benattar, F. Moussa, M. Lambert, J. Chim. Phys. 80, 99 (1983).

[5] B.I. Halperin, D.R. Nelson, Phys. Rev. Lett. 41, 121 (1978); A.N. Berker, D.R. Nelson, Phys. Rev. B 19, 2488 (1979).

[6] J.M. Kosterlitz, D.J. Thouless, J. Phys. C 6, 1181 (1973).

[7] T. Stoebe, C.C. Huang, J.W. Goodby, Phys. Rev. Lett. 68, 2944 (1992).

[8] A.J. Jin, M. Veum, T. Stoebe, C.F. Chou, J.T. Ho, S.W. Hui, V. Surendranath, C.C. Huang, Phys. Rev. Lett. 74, 4863 (1995).

[9] E. Gorecka, L. Chen, W. Pyzuk, A. Krowczynski, S. Kumar, Phys. Rev. E 50, 2863 (1994).

[10] Kh. El Guermai, M. Ayadi, J. Phys. IV 8, 47 (1998).

[11] G. Pepy, R. Fouret, M. More, L. Rosta, Phys. Scr. 39, 485 (1989).

[12] G. Pepy, R. Fouret, M. More, L. Rosta, Liq. Crystals 5, 571 (1989).

[13] M. More, C. Gors, P. Derollez, J. Matavar, Liq. Crystals 18, 337 (1995).

[14] G. Odou, D. Naviez, Spectra 2000 17, 73 (1987).

[15] A. Guinier, Théorie et technique de la radiocristallographie, Dunod, Paris 1956, p. 277.

[16] A.J. Leadbetter, K. Norris, Mol. Phys. 38, 669 (1979).

[17] P. Davidson, A.M. Levelut, J. Phys. (France) 50, 2415 (1989).

[18] J.J. Benattar, Ph.D. thesis, Université d'Orsay, Paris 1983. 